doi: $10.2306 /$ scienceasia1513-1874.2022.027

\title{
Meromorphic solutions of some types of $q$-difference differential equation and delay differential equation
}

\author{
Minfeng Chen ${ }^{\mathrm{a}, *}$, Zongsheng Gao ${ }^{\mathrm{b}}$, Jilong Zhang ${ }^{\mathrm{b}}$ \\ a School of Mathematics and Statistics, Guangdong University of Foreign Studies, Guangzhou 510006 China \\ b LMIB \& School of Mathematical Sciences, Beihang University, Beijing 100191 China
}

${ }^{*}$ Corresponding author, e-mail: chenminfeng198710@126.com

Received 8 May 2021

Accepted 10 Nov 2021

ABSTRACT: In this paper, we investigate the existence of rational solutions and value distribution of non-rational meromorphic solutions with the finite order of delay differential equation

$$
w(z+1)-w(z-1)+a \frac{w^{\prime}(z)}{w(z)}=b
$$

where $a \in \mathbb{C} \backslash\{0\}, b \in \mathbb{C}$ are constants. In addition, necessary conditions are obtained for $q$-difference differential equation

$$
w(q z)-w(z / q)+a(z) \frac{w^{\prime}(z)}{w(z)}=R(z, w(z))
$$

to admit a non-rational meromorphic solution of zero-order, where $|q| \notin\{0,1\}, a(z)$ is rational and $R(z, w(z))$ is rational in $w(z)$ with rational coefficients in $z$.

KEYWORDS: value distribution, meromorphic solution, delay differential equation, $q$-difference differential equation, zero-order

MSC2010: 30D05 34M10 39A45 39B32

\section{INTRODUCTION AND MAIN RESULTS}

Quispel et al [1] have derived the delay differential equation

$$
w(z+1)-w(z-1)+a \frac{w^{\prime}(z)}{w(z)}=b,
$$

where $a, b$ are constants, as a reduction of the integrable Kac-van Moerbeke equation on the basis of its Lie symmetries. They showed that (1) admits a Lax representation, and reduces to the first Painlevé equation in a continuum limit.

In this paper, we continue to study (1) and obtain some properties of meromorphic solutions of (1), that is, we obtain the following result.

Theorem 1 Consider the delay differential (1), where $a \in \mathbb{C} \backslash\{0\}, b \in \mathbb{C}$ are constants. Then

(i) If $b \in \mathbb{C} \backslash\{0\}$, then (1) has no rational solution;

(ii) If $b=0$, then (1) admits a non-constant rational solution of the form

$$
w(z)=-\frac{a}{2}+\frac{m(z)}{n(z)},
$$

where $m(z)$ and $n(z)$ are polynomials with $\operatorname{deg} m(z)=m, \operatorname{deg} n(z)=n$ and $m<n ;$

(iii) If $b \in \mathbb{C} \backslash\{0\}$, suppose that $w(z)$ is a transcendental meromorphic solution of (1) with finite order, then $w(z)$ has at most one finite Borel exceptional value 0 unless

$$
w(z)=p \exp (k \pi i z) \quad \text { and } \quad \frac{b}{a}=k \pi i, \quad i=\sqrt{-1},
$$

where $p$ is a non-zero constant, $k \in \mathbb{Z} \backslash\{0\} ;$

(iv) If $b \in \mathbb{C} \backslash\{0\}$, suppose that $w(z)$ is a finite order transcendental meromorphic solution of (1), and has only finitely many zeros and poles, then

$$
w(z)=p \exp (k \pi i z) \quad \text { and } \quad \frac{b}{a}=k \pi i, \quad i=\sqrt{-1},
$$

where $p$ is a non-zero constant, $k \in \mathbb{Z} \backslash\{0\}$.

Example $1([1])$

$$
\begin{aligned}
w(z) & =-\frac{a}{2} \frac{(z+c+1)(z+c-2)}{(z+c)(z+c-1)} \\
& =-\frac{a}{2}+\frac{a}{(z+c)(z+c-1)}
\end{aligned}
$$

is a rational solution of equation

$$
w(z+1)-w(z-1)+a \frac{w^{\prime}(z)}{w(z)}=0,
$$

where $a \in \mathbb{C} \backslash\{0\}$ and $c \in \mathbb{C}$. 
The difference analogue of the logarithmic derivative lemma, which was obtained independently by Halburd et al [2] and by Chiang et al [3], plays a key role in the value distribution of difference [4-9]. Also, $q$-difference analogue of the logarithmic derivative lemma, which was obtained by Barnett et al [10], plays an important role in the value distribution of $q$ difference [11-14].

Halburd et al [15] investigated the differentialdifference equation

$$
w(z+1)-w(z-1)+a(z) \frac{w^{\prime}(z)}{w(z)}=R(z, w(z)),
$$

where $a(z)$ is rational and $R(z, w(z))$ is rational in $w(z)$ and meromorphic in $z$. In this paper, we study $q$ difference differential equation

$$
\begin{aligned}
w(q z)-w(z / q)+a(z) \frac{w^{\prime}(z)}{w(z)} & =R(z, w(z)) \\
& =\frac{P(z, w(z))}{Q(z, w(z))},
\end{aligned}
$$

where $|q| \notin\{0,1\}, a(z)$ is rational and $R(z, w(z))$ is rational in $w(z)$ with rational coefficients in $z$. Now, we state the main findings as follows.

Theorem 2 Let $w(z)$ be a transcendental meromorphic solution of zero-order of (3), where $a(z)$ is rational in $z$, $P(z, w(z))$ is a polynomial in $w(z)$ having rational coefficients in $z$, and $Q(z, w(z)) \not \equiv 0$ is a monic polynomial in $w(z)$ with roots that are rational in $z$ and not roots of $P(z, w(z))$. Then

$$
\operatorname{deg}_{w}(P(z, w))=\operatorname{deg}_{w}(Q(z, w))+1 \leqslant 3,
$$

or the degree of $R(z, w(z))$ as a rational function in $w(z)$ is either 0 or 1 .

Considering a meromorphic function $w(z)$ in the complex plane, we assume that the reader is familiar with the standard symbols and fundamental results of Nevanlinna theory $[16,17]$. In addition, we use notations $\rho(w), \lambda(w)$ and $\lambda\left(\frac{1}{w}\right)$ to denote the order of growth, the exponent of convergence of the zerosequence and the pole-sequence of meromorphic function $w(z)$, respectively. And we denote by $S(r, w)$ any quantify satisfying $S(r, w)=o(T(r, w))$, as $r \rightarrow \infty$, outside of a possible exceptional set of finite logarithmic measure.

\section{LEMMAS}

Lemma 1 ([10], Theorem 1.2) Let $f(z)$ be a nonconstant zero-order meromorphic function, and $q \in$ $\mathbb{C} \backslash\{0\}$. Then

$$
m\left(r, \frac{f(q z)}{f(z)}\right)=o(T(r, f))
$$

on a set of logarithmic density 1 .
Lemma 2 ([14], Theorem 1.1) Let $f(z)$ be a nonconstant zero-order meromorphic function, and $q \in$ $\mathbb{C} \backslash\{0\}$. Then

$$
T(r, f(q z))=(1+o(1)) T(r, f(z))
$$

on a set of logarithmic density 1.

Remark 1 Equation (6) implies that

$$
T(r, f(q z))=T(r, f(z))+S(r, f) .
$$

Lemma 3 ([18], Lemma 4) Let $c_{1}>1, c_{2}>1$ and $\rho \geqslant$ 0. If $T: \mathbb{R}^{+} \rightarrow \mathbb{R}^{+}$is an increasing function such that

$$
\limsup _{r \rightarrow \infty} \frac{\log T(r)}{\log r}=\rho,
$$

then the logarithmic density of the set

$$
E:=\left\{r: T\left(c_{1} r\right) \geqslant c_{2} T(r)\right\}
$$

satisfies

$$
\overline{\log \operatorname{dens}}(E) \leqslant \frac{\rho \log c_{1}}{\log c_{2}} .
$$

The following lemma plays a key role in the proof of Theorem 2. A q-difference differential polynomial in $w(z)$ is defined by

$$
\begin{aligned}
P(z, w(z))= & \sum_{\lambda \in I} a_{\lambda}(z) w(z)^{\lambda_{0,0}} w\left(q_{1} z\right)^{\lambda_{1,0}} \cdots \\
& \cdots w\left(q_{v} z\right)^{\lambda_{\nu, 0}} w^{\prime}(z)^{\lambda_{0,1}} \cdots w^{(\mu)}\left(q_{v} z\right)^{\lambda_{v, \mu}},
\end{aligned}
$$

where $q_{1}, \ldots, q_{v}$ are distinct complex constants and $\left|q_{i}\right| \notin\{0,1\}(1 \leqslant i \leqslant v), I$ is a finite index set consisting of elements of the form $\lambda=\left(\lambda_{0,0}, \cdots, \lambda_{v, \mu}\right)$ and the coefficients $a_{\lambda}(z)$ are rational functions of $z$ for all $\lambda \in I$.

Lemma 4 Let $w(z)$ be a transcendental meromorphic solution of

$$
P(z, w(z))=0,
$$

where $P(z, w(z))$ is q-difference differential polynomial in $w(z)$ with rational coefficients, and let $b_{1}, \ldots, b_{l}$ be rational functions satisfying $P\left(z, b_{j}\right) \not \equiv 0$ for all $j \in$ $\{1, \ldots, l\}$. Denote $K=\max _{1 \leqslant i \leqslant v}\left\{\left|q_{i}\right|, 1 /\left|q_{i}\right|\right\}$. If there exists $s>0$ and $\tau \in(0,1)$ such that

$$
\sum_{j=1}^{l} n\left(r, \frac{1}{w-b_{j}}\right) \leqslant l \tau n\left(K^{s} r, w\right)+O(1),
$$

then $\rho(w)>0$.

Proof: Suppose that $\rho(w)=0$. We first show that the assumption $P\left(z, b_{j}\right) \not \equiv 0$ implies that

$$
m\left(r, \frac{1}{w-b_{j}}\right)=S(r, w),
$$


on a set of logarithmic density 1 . This result is an extension of Mohon'ko's Theorem [19] and its $q$-difference analogue [10] for differential-difference equations with meromorphic solutions of zero-order.

By substituting $w=g+b_{j}$ into (7) we obtain

$$
Q(z, g)+R(z)=0 \text {, }
$$

where

$$
Q(z, g)=\sum_{\lambda \in I} a_{\lambda}(z) G_{\lambda}(z, g)
$$

is a $q$-difference differential polynomial in $g(z)$ such for all $\lambda$ in the finite index set $I, G_{\lambda}(z, g)$ is a non-constant product of derivatives and $q$-shift of $g(z)$. Also $R(z) \not \equiv$ 0 , since $P\left(z, b_{j}\right) \not \equiv 0$ for all $j \in\{1, \ldots, l\}$, is a rational function. The coefficients $a_{\lambda}(z)$ in (11) are all rational. By defining $E_{1}=\left\{\theta \in[0,2 \pi):\left|g\left(r \mathrm{e}^{i \theta}\right)\right| \leqslant 1\right\}$ and $E_{2}=$ $[0,2 \pi) \backslash E_{1}$, we have

$$
\begin{aligned}
m\left(r, \frac{1}{w-b_{j}}\right) & =m\left(r, \frac{1}{g}\right) \\
& =\int_{\theta \in E_{1}} \log ^{+}\left|\frac{1}{g\left(r \mathrm{e}^{i \theta}\right)}\right| \frac{d \theta}{2 \pi} .
\end{aligned}
$$

Furthermore, for all $z=r \mathrm{e}^{i \theta}$ such that $\theta \in E_{1}$,

$$
\begin{aligned}
\left|\frac{Q(z, g)}{g}\right|= & \frac{1}{|g|} \mid \sum_{\lambda \in I} a_{\lambda}(z) g(z)^{\lambda_{0,0}} g\left(q_{1} z\right)^{\lambda_{1,0}} \ldots \\
& \cdots g\left(q_{v} z\right)^{\lambda_{v, 0}} g^{\prime}(z)^{\lambda_{0,1}} \cdots g^{(\mu)}\left(q_{v} z\right)^{\lambda_{v, \mu}} \mid \\
\leqslant & \left.\sum_{\lambda \in I}\left|a_{\lambda}(z)\right| \frac{g\left(q_{1} z\right)}{g(z)}\right|^{\lambda_{1,0}} \cdots \\
& \ldots\left|\frac{g\left(q_{v} z\right)}{g(z)}\right|^{\lambda_{v, 0}}\left|\frac{g^{\prime}(z)}{g(z)}\right|^{\lambda_{0,1}} \cdot\left|\frac{g^{(\mu)}\left(q_{v} z\right)}{g(z)}\right|^{\lambda_{v, \mu}},
\end{aligned}
$$

since $\operatorname{deg}_{g}\left(G_{\lambda}\right) \geqslant 1$ for all $\lambda \in I$ with $\lambda=$ $\left(\lambda_{0,0}, \ldots, \lambda_{v, \mu}\right)$. Using (10) we have

$$
\begin{aligned}
\log ^{+}\left|\frac{1}{g(z)}\right| & \leqslant \log ^{+}\left|\frac{R(z)}{g(z)}\right|+\log ^{+}\left|\frac{1}{R(z)}\right| \\
& \leqslant \log ^{+}\left|\frac{Q(z, g)}{g(z)}\right|+\log ^{+}\left|\frac{1}{R(z)}\right| .
\end{aligned}
$$

By using (12) with $q_{0}=1$, and applying the lemma on the logarithmic derivative, Lemma 1 and Lemma 2, we have

$$
\begin{aligned}
m\left(r, \frac{1}{w-b_{j}}\right) & \leqslant \int_{\theta \in E_{1}} \log ^{+}\left|\frac{Q(z, g)}{g\left(r e^{i \theta}\right)}\right| \frac{d \theta}{2 \pi}+O(\log r) \\
& \leqslant \sum_{n=0}^{v} \sum_{m=0}^{\mu} \lambda_{n, m} m\left(r, \frac{g^{(m)}\left(q_{n} z\right)}{g(z)}\right)+O(\log r) \\
& \leqslant \sum_{n=0}^{v} \sum_{m=0}^{\mu} \lambda_{n, m}\left(m\left(r, \frac{g^{(m)}\left(q_{n} z\right)}{g\left(q_{n} z\right)}\right)\right. \\
+ & \left.m\left(r, \frac{g\left(q_{n} z\right)}{g(z)}\right)\right)+O(\log r)=S(r, w),
\end{aligned}
$$

on a set of logarithmic density 1. It follows from (8) that

$$
\sum_{j=1}^{l} N\left(r, \frac{1}{w-b_{j}}\right) \leqslant l(\tau+\varepsilon) N\left(K^{s} r, w\right)+O(\log r),
$$

where $\varepsilon>0$ is chosen such that $\tau+\varepsilon<1$. By the first main theorem of Nevanlinna theory, we have

$$
l T(r, w)=\sum_{j=1}^{l}\left(m\left(r, \frac{1}{w-b_{j}}\right)+N\left(r, \frac{1}{w-b_{j}}\right)\right)+O(\log r) .
$$

It follows from (9), (14) and (15) that

$$
\begin{aligned}
l T(r, w) & \leqslant l(\tau+\varepsilon) N\left(K^{s} r, w\right)+S(r, w) \\
& \leqslant l(\tau+\varepsilon) T\left(K^{s} r, w\right)+S(r, w) .
\end{aligned}
$$

By Remark 1 and an observation due to Bergweiler et al [20], it follows that

$T\left(K^{s} r, w(z)\right)+O(1)=T\left(r, w\left(K^{s} z\right)\right)=T(r, w)+S(r, w)$

on a set of logarithmic density 1. By combining (16), we have

$$
T(r, w) \leqslant(\tau+\varepsilon) T(r, w)+S(r, w),
$$

since $\tau+\varepsilon<1$, the above inequality is a contradiction. Then we conclude that $\rho(w)>0$.

\section{PROOF OF Theorem 1}

Proof: Suppose that $w(z)$ is a rational solution of (1). Denote

$$
w(z)=h(z)+\frac{m(z)}{n(z)},
$$

where $h(z), m(z)$ and $n(z)$ are polynomial with $\operatorname{deg} h(z)=l, \operatorname{deg} m(z)=m, \operatorname{deg} n(z)=n$ with $m<n$. Set

$$
\begin{aligned}
h(z) & =c_{0} z^{l}+\cdots+c_{l}, \\
m(z) & =a_{0} z^{m}+\cdots+a_{m}, \\
n(z) & =b_{0} z^{n}+\cdots+b_{n},
\end{aligned}
$$

where $c_{0}, \ldots, c_{l}, a_{0}(\neq 0), \ldots, a_{m}, b_{0}(\neq 0), \cdots, b_{n}$ are constants.

(i) If $b \in \mathbb{C} \backslash\{0\}$, in what follows, we consider three cases.

Case 1: $l>0$. By (17) and (18), when $z$ is large enough, $w(z)$ can be written as

$$
w(z)=c_{0} z^{l}(1+o(1))
$$

Then

$$
\begin{aligned}
& w(z+1)=c_{0}(z+1)^{l}(1+o(1)), \\
& w(z-1)=c_{0}(z-1)^{l}(1+o(1)), \\
& w(z+1)-w(z-1)=2 c_{0} l z^{l-1}(1+o(1)), \\
& w^{\prime}(z)=c_{0} l z^{l-1}(1+o(1)) .
\end{aligned}
$$


By substituting (19) and (20) into (1), it follows that

$$
\begin{array}{r}
c_{0} z^{l}(1+o(1)) 2 c_{0} l z^{l-1}(1+o(1))+a c_{0} l z^{l-1}(1+o(1)) \\
=b c_{0} z^{l}(1+o(1)),
\end{array}
$$

that is,

$$
2 c_{0}^{2} l z^{2 l-1}(1+o(1))=b c_{0} z^{l}(1+o(1)),
$$

from which it follows that

$$
2 l-1=l \text { and } 2 c_{0}^{2}=b c_{0} .
$$

So $l=1$ and $b=2 c_{0}$. Then $w(z)$ can be rewritten as

$$
w(z)=c_{0} z+c_{1}+o(1), \quad c_{0} \neq 0,
$$

and

$$
\begin{aligned}
& \begin{array}{l}
w(z+1)-w(z-1) \\
\quad=c_{0}(z+1)+c_{1}+o(1)-\left[c_{0}(z-1)+c_{1}+o(1)\right] \\
\quad=2 c_{0}+o(1),
\end{array} \\
& \begin{array}{l}
w^{\prime}(z)=c_{0}+o(1) .
\end{array}
\end{aligned}
$$

Substituting (21) and (22) into (1) yields

$$
\begin{aligned}
\left(c_{0} z+c_{1}+o(1)\right)\left(2 c_{0}+o(1)\right)+a( & \left.c_{0}+o(1)\right) \\
& =b\left(c_{0} z+c_{1}+o(1)\right) .
\end{aligned}
$$

Since $b=2 c_{0}$, from the above equality, we conclude that $a=0$, a contradiction.

Case 2: $l=0, c_{0} \neq 0$. By (17) and (18), when $z$ is large enough, $w(z)$ can be written as

$$
w(z)=c_{0}+\frac{m(z)}{n(z)}=c_{0}+o(1) .
$$

By calculation and $m<n$, we see that

$$
\begin{aligned}
& m(z+1) n(z-1)-m(z-1) n(z+1) \\
& \quad=2(m-n) a_{0} b_{0} z^{m+n-1}(1+o(1)), \\
& \begin{aligned}
m^{\prime}(z) n(z)- & m(z) n^{\prime}(z) \\
& =(m-n) a_{0} b_{0} z^{m+n-1}(1+o(1)),
\end{aligned} \\
& n(z+1) n(z-1)=b_{0}^{2} z^{2 n}(1+o(1)), \\
& n^{2}(z)=b_{0}^{2} z^{2 n}(1+o(1)) .
\end{aligned}
$$

Then

$$
\begin{aligned}
w(z+1) & -w(z-1) \\
& =\frac{m(z+1) n(z-1)-m(z-1) n(z+1)}{n(z+1) n(z-1)} \\
& =2(m-n) \frac{a_{0}}{b_{0}} z^{m-n-1}(1+o(1)), \\
w^{\prime}(z) & =\frac{m^{\prime}(z) n(z)-m(z) n^{\prime}(z)}{n^{2}(z)} \\
& =(m-n) \frac{a_{0}}{b_{0}} z^{m-n-1}(1+o(1)) .
\end{aligned}
$$

Substituting (23)-(25) into (1) yields

$$
\begin{aligned}
\left(c_{0}+o(1)\right) 2(m-n) & \frac{a_{0}}{b_{0}} z^{m-n-1}(1+o(1))+ \\
a(m-n) & \frac{a_{0}}{b_{0}} z^{m-n-1}(1+o(1))=b\left(c_{0}+o(1)\right),
\end{aligned}
$$

that is,

$$
\left(a+2 c_{0}\right)(m-n) \frac{a_{0}}{b_{0}} z^{m-n-1}(1+o(1))=b\left(c_{0}+o(1)\right) .
$$

Since $m<n$ and $b, c_{0} \neq 0$, the above equality is a contradiction.

Case 3: $l=0, c_{0}=0$. Because $m<n$, we see that

$$
w(z)=\frac{m(z)}{n(z)}=\frac{a_{0}}{b_{0}} z^{m-n}(1+o(1)) .
$$

By substituting (24)-(26) into (1), we have

$$
\begin{aligned}
& \frac{a_{0}}{b_{0}} z^{m-n}(1+o(1)) 2(m-n) \frac{a_{0}}{b_{0}} z^{m-n-1}(1+o(1)) \\
& +a(m-n) \frac{a_{0}}{b_{0}} z^{m-n-1}(1+o(1))=b \frac{a_{0}}{b_{0}} z^{m-n}(1+o(1)),
\end{aligned}
$$

that is,

$$
a(m-n) \frac{a_{0}}{b_{0}} z^{m-n-1}(1+o(1))=b \frac{a_{0}}{b_{0}} z^{m-n}(1+o(1)) .
$$

Since $m<n$ and $a b \neq 0$, the above equality is a contradiction.

(ii) If $b=0$, suppose that $l>0$ or $l=0$ and $c_{0}=0$, we can conclude that (1) has no non-constant rational solution by using the same method of proof of Case 1 or Case 3 , respectively. Suppose that $l=0$ and $c_{0} \neq 0$, substitute (23)-(25) into (1) yields

$$
\begin{aligned}
\left(c_{0}+o(1)\right) 2(m-n) & \frac{a_{0}}{b_{0}} z^{m-n-1}(1+o(1))+ \\
& a(m-n) \frac{a_{0}}{b_{0}} z^{m-n-1}(1+o(1))=0,
\end{aligned}
$$

that is,

$$
\left(a+2 c_{0}\right)(m-n) \frac{a_{0}}{b_{0}} z^{m-n-1}(1+o(1))=0 .
$$

Since $m<n$ and $a_{0}, b_{0} \neq 0$, then we have $c_{0}=-\frac{a}{2}$.

(iii) If $b \in \mathbb{C} \backslash\{0\}$, denote

$P(z, w):=w(z)[w(z+1)-w(z-1)]+a w^{\prime}(z)-b w(z)=0$.

Then, we have

$$
P(z, \alpha)=-b \alpha \neq 0, \quad \alpha \in \mathbb{C} \backslash\{0\} .
$$

An application of the estimate (2.3) of Lemma 2.1 in [15], yields

$$
m\left(r, \frac{1}{w-\alpha}\right)=S(r, w) .
$$


Then

$$
N\left(r, \frac{1}{w-\alpha}\right)=T(r, w)+S(r, w)
$$

for all $\alpha \in \mathbb{C} \backslash\{0\}$. Thus $w(z)$ has at most one finite Borel exceptional value 0 . Suppose that $w(z)$ has two Borel exceptional values 0 and $\infty$, by Theorem 2.11 of [21], we see that $w(z)$ is of regular growth, then $w(z)$ can be written as

$$
w(z)=p(z) \mathrm{e}^{h(z)}
$$

where $p(z)$ is a meromorphic function, and $h(z)$ is polynomial such that

$$
\sigma(p(z))=\max \{\lambda(w), \lambda(1 / w)\}<\operatorname{deg} h(z) .
$$

By (27), we obtain

$$
\begin{aligned}
w(z+1) & =p(z+1) \mathrm{e}^{h(z+1)}=p_{+}(z) \mathrm{e}^{h(z)}, \\
w(z-1) & =p(z-1) \mathrm{e}^{h(z-1)}=p_{-}(z) \mathrm{e}^{h(z)}, \\
w^{\prime}(z) & =\left(p^{\prime}(z)+p(z) h^{\prime}(z)\right) \mathrm{e}^{h(z)},
\end{aligned}
$$

where $p_{+}(z)=p(z+1) \mathrm{e}^{h(z+1)-h(z)}, \quad p_{-}(z)=$ $p(z-1) \mathrm{e}^{h(z-1)-h(z)}$. Substituting (27) and (28) into (1) yields

$$
\begin{array}{r}
p(z)\left(p_{+}(z)-p_{-}(z)\right) \mathrm{e}^{h(z)} \\
=b p(z)-a\left(p^{\prime}(z)+p(z) h^{\prime}(z)\right) .
\end{array}
$$

If $p_{+}(z)-p_{-}(z) \not \equiv 0$, rewrite (29) as

$$
\mathrm{e}^{h(z)}=\frac{b p(z)-a\left(p^{\prime}(z)+p(z) h^{\prime}(z)\right)}{p(z)\left(p_{+}(z)-p_{-}(z)\right)} .
$$

Since $\sigma\left(e^{h(z)}\right)=\operatorname{deg} h(z)$ and

$$
\begin{aligned}
& \sigma\left(\frac{b p(z)-a\left(p^{\prime}(z)+p(z) h^{\prime}(z)\right)}{p(z)\left(p_{+}(z)-p_{-}(z)\right)}\right) \\
& \leqslant \max \{\operatorname{deg} h(z)-1, \sigma(p(z))\}
\end{aligned}
$$

which contradicts with $\sigma(p(z))<\operatorname{deg} h(z)$. Thus $p_{+}(z)-p_{-}(z) \equiv 0$, then $a\left(p^{\prime}(z)+p(z) h^{\prime}(z)\right)-b p(z) \equiv 0$. By $p(z) \not \equiv 0$ and $a\left(p^{\prime}(z)+p(z) h^{\prime}(z)\right)-b p(z) \equiv 0$, it follows that $p^{\prime}(z) / p(z) \equiv b / a-h^{\prime}(z)$, that is, $p(z)=$ $c \mathrm{e}^{b z / a-h(z)}$, where $c$ is a non-zero constant. Since $1 \leqslant \operatorname{deg} h(z)$ and $\sigma(p(z))<\operatorname{deg} h(z)$, we have $h(z)=$ $b z / a+c_{1}$ and $p(z) \equiv p$, where $p$ is a non-zero constant, $c_{1}$ is a constant. It follows from $p_{+}(z)-p_{-}(z) \equiv 0$ and $p(z) \equiv p$ that $p(z+1) \mathrm{e}^{h(z+1)-h(z)} \equiv p(z-1) e^{h(z-1)-h(z)}$, then $\mathrm{e}^{2 b / a}=1$, that is, $b / a=k \pi i, k \in \mathbb{Z} \backslash\{0\}$. So $w(z)=p \exp (k \pi i z)$.

(iv) Suppose that $w(z)$ has only finitely many zeros and poles, and is a finite order transcendental meromorphic solution of (1), then 0 and $\infty$ are two Borel exceptional values of $w(z)$, the conclusion follows immediately by Theorem 1 (iii).

\section{PROOF OF Theorem 2}

Proof: Suppose that (3) has a transcendental meromorphic solution of zero-order. By (3) and an identity due to Valiron [22] and Mohon'ko [23] (see also [17], Theorem 2.2.5), we have

$$
\begin{aligned}
T(r, w(q z) & \left.-w(z / q)+a(z) \frac{w^{\prime}(z)}{w(z)}\right) \\
= & T(r, R(z, w(z))) \\
= & \operatorname{deg}_{w}(R(z, w(z))) T(r, w(z))+O(\log r) .
\end{aligned}
$$

By applying the lemma on the logarithmic derivative and an observation due to Bergweiler et al [20], we have for all sufficiently large $r>0$,

$$
\begin{aligned}
& \operatorname{deg}_{w}(R(z, w(z))) T(r, w(z)) \\
& \quad \leqslant T(r, w(q z))+T(r, w(z / q))+T\left(r, \frac{w^{\prime}(z)}{w(z)}\right)+O(\log r) \\
& \quad \leqslant T(r, w(q z))+T\left(r, w\left(\frac{z}{q}\right)\right)+\bar{N}(r, w)+\bar{N}\left(r, \frac{1}{w}\right)+S(r, w) \\
& \quad \leqslant T(|q| r, w(z))+T\left(\frac{r}{|q|}, w(z)\right)+2 T(r, w(z))+S(r, w) \\
& \quad \leqslant(2+\varepsilon) T(K r, w(z))+2 T(r, w(z)),
\end{aligned}
$$

that is,

$$
\begin{aligned}
\left(\operatorname{deg}_{w}(R(z, w(z)))-2\right) T( & r, w(z)) \\
& \leqslant(2+\varepsilon) T(K r, w(z)),
\end{aligned}
$$

with $K=\max \{|q|, 1 /|q|\}$ and $\varepsilon>0$. Let

$$
G=\{r>0 \text { : inequality (30) is true }\} .
$$

Then the logarithmic density of $G$ satisfies

$$
\overline{\log \operatorname{dens}}(G)=\limsup _{r \rightarrow \infty} \frac{\int[0, r] \cap G \frac{d t}{t}}{\log r}=1 .
$$

By Lemma 3 and inequality (30), it follows that

$$
\overline{\log \operatorname{dens}}(G) \leqslant \frac{\rho(w) \log K}{\log \left(\frac{\operatorname{deg}_{w}(R(z, w(z)))-2}{2+\varepsilon}\right)},
$$

and so

$$
\log \left(\frac{\operatorname{deg}_{w}(R(z, w(z)))-2}{2}\right) \leqslant \rho(w) \log K,
$$

by letting $\varepsilon \rightarrow 0$. Hence, if (3) has a non-rational meromorphic solution of zero-order, then it follows from (31) that

$$
\operatorname{deg}_{w}(R(z, w(z))) \leqslant 4 .
$$

In what follows, similar to the proof of Theorem 1.1 in [15], we consider five cases.

Case 1: Suppose that the denominator of $R(z, w(z))$ has at least two distinct non-zero rational 
roots for $w(z)$ as a function of $z$, say $b_{1}(z) \not \equiv 0$ and $b_{2}(z) \not \equiv 0$. Then we written (3) as

$$
\begin{aligned}
w(q z)- & w(z / q)+a(z) \frac{w^{\prime}(z)}{w(z)}= \\
& \frac{P(z, w(z))}{\left(w(z)-b_{1}(z)\right)\left(w(z)-b_{2}(z)\right) \hat{Q}(z, w(z))},
\end{aligned}
$$

where $P(z, w(z)) \not \equiv 0$ and $\hat{Q}(z, w(z)) \not \equiv 0$ are polynomials in $w(z)$ of at most degree 4 and 2 , respectively. We do not rule out the possibility that $\hat{Q}\left(z, b_{1}(z)\right) \equiv 0$ or $\hat{Q}\left(z, b_{2}(z)\right) \equiv 0$. We also assume that $P(z, w(z))$ and $\hat{Q}(z, w(z))$ are two mutually prime polynomials in $w(z)$. Then neither $b_{1}(z)$, nor $b_{2}(z)$ is a solution of (32), and so they satisfy the first condition of Lemma 4. Assume that $\hat{z} \in \mathbb{C}$ is any point satisfying

$$
w(\hat{z})=b_{1}(\hat{z})
$$

and such that none of the coefficients of (32) have a zero or pole at $\hat{z}$ and $P(\hat{z}, w(\hat{z})) \neq 0$. Let $p$ denote the order of the zero of $w(z)-b_{1}(z)$ at $\hat{z}$. We call such a $\hat{z}$ a generic root of $w(z)-b_{1}(z)$ of order $p$. Without further comment, we only consider generic roots. Since the coefficients are rational and thus have finitely many zeros or poles, the contribution can be included in a bounded error term, leading to an error term of the type $O(\log r)$. It follows from (32) that either $w(q z)$ or $w(z / q)$ has a pole at $z=\hat{z}$ of order at least $p$. Without loss of generality, we assume that $w(q z)$ has such a pole at $z=\hat{z}$. By $q$-shifting the (32), we have

$$
\begin{aligned}
& w\left(q^{2} z\right)-w(z)+a(q z) \frac{q w^{\prime}(q z)}{w(q z)}= \\
& \frac{P(q z, w(q z))}{\left(w(q z)-b_{1}(q z)\right)\left(w(q z)-b_{2}(q z)\right) \hat{Q}(q z, w(q z))},
\end{aligned}
$$

which implies that $w\left(q^{2} z\right)$ has such a pole of order one at $z=\hat{z}$ provided that

$$
\operatorname{deg}_{w}(P) \leqslant \operatorname{deg}_{w}(\hat{Q})+2 .
$$

Suppose that (35) holds, by iterating (32) one more step, we have

$$
\begin{aligned}
& w\left(q^{3} z\right)-w(q z)+a\left(q^{2} z\right) \frac{q^{2} w^{\prime}\left(q^{2} z\right)}{w\left(q^{2} z\right)}= \\
& \frac{P\left(q^{2} z, w\left(q^{2} z\right)\right)}{\left(w\left(q^{2} z\right)-b_{1}\left(q^{2} z\right)\right)\left(w\left(q^{2} z\right)-b_{2}\left(q^{2} z\right)\right) \hat{Q}\left(q^{2} z, w\left(q^{2} z\right)\right)} .
\end{aligned}
$$

If $p>1$, then $w\left(q^{3} z\right)$ must have a pole of order at least $p$ at $z=\hat{z}$. Hence, we can pair up the zero of $w(z)-b_{1}(z)$ at $z=\hat{z}$ together with the pole of $w(z)$ at $z=q \hat{z}$ without possibility of a similar iteration process starting from another point, say $z=q^{3} \hat{z}$, and resulting in pairing the pole at $z=q \hat{z}$ with another root of $w(z)-b_{1}(z)$, or of $w(z)-b_{2}(z)$. Therefore, we have found a pole of order at least $p$ which can be uniquely associated with the zero of $w(z)-b_{1}(z)$ at $z=\hat{z}$. If $p=1$, it may in principle be possible that there is another root of $w(z)-b_{1}(z)$ or of $w(z)-b_{2}(z)$ at $z=q^{3} \hat{z}$, which needs to be paired with the pole of $w(z)$ at $z=q^{2} \hat{z}$. But since now all of the poles in the iteration are simple, we still pair up the root of $w(z)$ at $z=\hat{z}$ and the pole of $w(z)$ at $z=q \hat{z}$. If there is another root of $w(z)-b_{1}(z)$ at $z=q^{3} \hat{z}$ such that $w\left(q^{4} \hat{z}\right)$ is finite, we can pair it up with pole of $w(z)$ at $z=q^{2} \hat{z}$. Thus for any $p \geqslant 1$, there is a pole of order at least $p$ which can be paired up with the root of $w(z)-b_{1}(z)$ at $z=\hat{z}$. By adding up all points $\hat{z}$ such that (33) holds, similarly, for $b_{2}\left(\not \equiv b_{1}\right)$, it follows that

$n\left(r, \frac{1}{w-b_{1}}\right)+n\left(r, \frac{1}{w-b_{2}}\right) \leqslant n(K r, w)+O(1)$

with $K=\max \{|q|, 1 /|q|\}$. Hence, it satisfies the second condition of Lemma 4, so $\rho(w)>0$, a contradiction.

Suppose that

$$
\operatorname{deg}_{w}(P)>\operatorname{deg}_{w}(\hat{Q})+2 .
$$

If $\operatorname{deg}_{w}(P)=3$, it follows that $\operatorname{deg}_{w}(Q)=2$, and the assertion (4) holds. If

$$
4=\operatorname{deg}_{w}(P)>\operatorname{deg}_{w}(\hat{Q})+2=2,
$$

and $\hat{z}$ is a generic root of $w(z)-b_{1}(z)$ of order $p$. It follows from (32) that either $w(q z)$ or $w(z / q)$ must have a pole at $z=\hat{z}$ of order at least $p$, and we suppose as above that $w(q z)$ has the pole at $z=\hat{z}$. Then, it follows that $w\left(q^{2} \hat{z}\right)$ has a pole of order $2 p$, and $w\left(q^{3} \hat{z}\right)$ has a pole of order of $4 p$. Hence we can pair the root of $w(z)-b_{1}(z)$ at $z=\hat{z}$ and the pole of $w(z)$ at $z=q \hat{z}$ the same way as in the case (35). Similarly, for the roots of $w(z)-b_{2}(z)$, and so (37) holds. By Lemma 4, $\rho(w)>0$, a contradiction.

Suppose that

$$
4=\operatorname{deg}_{w}(P)>\operatorname{deg}_{w}(\hat{Q})+2=3,
$$

and that $\hat{z}$ is a point satisfying (33), and of order p. Since $\operatorname{deg}_{w}(\hat{Q})=1$, without loss of generality, we assume that

$$
\hat{Q}=w(z)-b_{3}(z),
$$

where $b_{3}(z)$ is rational in $z$. Assume that $b_{3}(z) \not \equiv b_{j}(z)$, for $j \in\{1,2\}$. It follows by the assumption $Q(z, w) \not \equiv 0$ that $b_{3}(z) \not \equiv 0$. As above, it follows by (32) that either $w(q z)$ or $w(z / q)$ has a pole of order at least $p$ at $z=\hat{z}$, and we may again suppose that $w(q z)$ has that pole. If $p>1$, then (34) implies that $w\left(q^{2} z\right)$ has a pole at $z=\hat{z}$ of order at least $p$. Even though, $w(z)-b_{j}(z)$ has a root at $z=q^{3} \hat{z}$ for some $j \in\{1,2,3\}$, we have found at least one pole for each root of $w(z)-b_{1}(z)$ in this 
iteration sequence, counting multiplicities. Therefore we can pair the root of $w(z)-b_{1}(z)$ at $z=\hat{z}$ and the pole of $w(z)$ at $z=q \hat{z}$ the same way as in (35) and (38). If $p=1$, it may in principle be possible that the pole of right hand side of (34) at $z=\hat{z}$ cancels with the pole of the term $a(q z) q w^{\prime}(q z) / w(q z)$ at $z=\hat{z}$ in such a way that $w\left(q^{2} \hat{z}\right)$ remains finite. If $w\left(q^{2} \hat{z}\right) \neq b_{j}\left(q^{2} \hat{z}\right)$ for $j \in\{1,2,3\}$, then it follows from (36) that $w\left(q^{3} z\right)$ has a pole at $z=\hat{z}$, and we can pair up the root of $w(z)-b_{1}(z)$ at $z=\hat{z}$ and the pole of $w(z)$ at $z=q \hat{z}$. If $w\left(q^{2} \hat{z}\right)=b_{j}\left(q^{2} \hat{z}\right)$ for $j \in\{1,2,3\}$, it may happen that $w\left(q^{3} \hat{z}\right)$ remains finite. If all points $\hat{z}$ such that $w(\hat{z})=$ $b_{j}(\hat{z})$ are a part of an iteration sequence of this form, i.e.,

$$
\begin{aligned}
& w(\hat{z})=b_{j_{1}}(\hat{z}), \quad w(q \hat{z})=\infty, \quad w(\hat{z})=b_{j_{2}}(\hat{z}), \\
& j_{1}, j_{2} \in\{1,2,3\} \text {, }
\end{aligned}
$$

by adding up all roots of $w(z)-b_{j}(z), j \in\{1,2,3\}$, we have

$n\left(r, \frac{1}{w-b_{1}}\right)+n\left(r, \frac{1}{w-b_{2}}\right)+n\left(r, \frac{1}{w-b_{3}}\right) \leqslant 2 n(K r, w)+O(1)$,

with $K=\max \{|q|, 1 /|q|\}$. Note that, all of $b_{1}, b_{2}$ and $b_{3}$ are not solutions of (32), it follows from Lemma 4 that $\rho(w)>0$, a contradiction.

Case 2: Suppose that the denominator of $R(z, w(z))$ in (3) has at least one non-zero rational root, say $b_{1}(z) \not \equiv 0$. Then (3) can be written as

$$
\begin{aligned}
& w(q z)-w(z / q)+a(z) \frac{w^{\prime}(z)}{w(z)}= \\
& \frac{P(z, w(z))}{\left(w(z)-b_{1}(z)\right)^{n} \breve{Q}(z, w(z))},
\end{aligned}
$$

where $P(z, w(z)) \not \equiv 0$ and $\left(w(z)-b_{1}(z)\right)^{n} Q(z, w(z))$ are two mutually prime polynomials in $w(z)$ with $\operatorname{deg}_{w}(P) \leqslant 4$ and $\operatorname{deg}_{w}\left(\left(w(z)-b_{1}(z)\right)^{n} Q(z, w(z))\right)=$ $n+\operatorname{deg}_{w}(Q)=n+m \leqslant 4$. Then $b_{1}(z)$ is not a solution of (40), and satisfies the first condition of Lemma 4. Assume that $n \in\{2,3,4\}$, and suppose that $\hat{z}$ is a generic root of $w(z)-b_{1}(z)$ of order $p$. Then either $w(q z)$ or $w(z / q)$ has a pole at $z=\hat{z}$ of order at least $n p$, and without loss of generality, we suppose that $w(q z)$ has such that a pole at $z=\hat{z}$. Next, suppose that

$$
\operatorname{deg}_{w}(P) \leqslant n+m
$$

Then $w\left(q^{2} \hat{z}\right)$ is a pole of order one, and $w\left(q^{3} \hat{z}\right)$ is a pole of order at least $n p$. By continuing the iteration, it follows that $w\left(q^{4} \hat{z}\right)$ is again a simple pole or finite value. Thus it may be $w\left(q^{4} \hat{z}\right)=b_{1}\left(q^{4} \hat{z}\right)$, and so it is at least in principle possible that $w\left(q^{5} \hat{z}\right)$ is a finite value. By adding up all roots of $w(z)-b_{1}(z)$ and poles of $w(z)$ in the set $\left\{\hat{z}, q \hat{z}, \cdots, q^{4} \hat{z}\right\}$, and taking into account multiplicities of these points, we find that there at least $2 n p+1$ poles for $2 p$ roots of $w(z)-b_{1}(z)$. If $w\left(q^{4} \hat{z}\right) \neq b_{1}\left(q^{4} \hat{z}\right)$, or $w\left(q^{4} \hat{z}\right)$ is a root of $\mathscr{Q}(z, w(z))$ with multiplicity $p$, then $w\left(q^{5} \hat{z}\right)$ is a pole of order $n p$, and we have even more poles for every root of $w(z)-b_{1}(z)$. By adding up the contribution from all points $z=\hat{z}$ to corresponding counting functions, it follows that

$$
n\left(r, \frac{1}{w-b_{1}}\right) \leqslant \frac{1}{n} n\left(K^{4} r, w\right)+O(1),
$$

with $K=\max \{|q|, 1 /|q|\}$. Then the second condition of Lemma 4 is satisfied, so $\rho(w)>0$, a contradiction.

Suppose that

$$
\operatorname{deg}_{w}(P)>n+m+1
$$

Suppose that $z=\hat{z}$ is a generic root of $w(z)-b_{1}(z)$ of order $p$. As in the case (41) either $w(q \hat{z})$ or $w(\hat{z} / q)$, say $w(q \hat{z})$, is a pole of order $n p$ at least. This implies that $w\left(q^{2} \hat{z}\right)$ is a pole of order $n p$ at least, and so, the only way that $w\left(q^{4} \hat{z}\right)$ can be finite is that $w\left(q^{3} \hat{z}\right)=b_{1}\left(q^{3} \hat{z}\right)$, or $w\left(q^{3} \hat{z}\right)$ is a root of $\mathscr{Q}(z, w(z))$, with multiplicity $p$. This is the "best case", we have found $2 n p$ poles, counting multiplicities, that correspond uniquely to $2 p$ roots of $w(z)-b_{1}(z)$. Thus, we have

$$
n\left(r, \frac{1}{w-b_{1}}\right) \leqslant \frac{1}{n} n\left(K^{3} r, w\right)+O(1),
$$

with $K=\max \{|q|, 1 /|q|\}$. Lemma 4 implies that $\rho(w)>0$, a contradiction.

Case 3: Suppose that $Q(z, w)$ in (3) has only one simple root, and assume that

$$
\operatorname{deg}_{w}(P) \geqslant 3
$$

Then the denominator of the right hand side of (3) can be written as $Q(z, w(z))=w(z)-b_{1}(z)$. Let $z=\hat{z}$ be a generic root of $w(z)-b_{1}(z)$ of order $p$. Then, either $w(q \hat{z})$ or $w(\hat{z} / q)$ is a pole of order $p$ at least. Without loss of generality, we assume that $w(q \hat{z})$ is a pole of order $p$. Then $w\left(q^{2} \hat{z}\right)$ is a pole of order $2 p$ at least, and $w\left(q^{3} \hat{z}\right)$ is a pole of order $4 p$ at least, and so on. Then we have

$$
n\left(r, \frac{1}{w-b_{1}}\right) \leqslant \frac{1}{3} n\left(K^{2} r, w\right)+O(1),
$$

with $K=\max \{|q|, 1 /|q|\}$. Lemma 4 implies that $\rho(w)>0$, a contradiction.

Case 4: Suppose that $Q(z, w)$ in (3) has only one simple root, and

$$
\operatorname{deg}_{w}(P) \leqslant 2 .
$$

If $\operatorname{deg}_{w}(P)=2$, then $\operatorname{deg}_{w}(P)=\operatorname{deg}_{w}(Q)+1$, and thus (4) holds. If $\operatorname{deg}_{w}(P) \leqslant 1$, then $\operatorname{deg}_{w}(R)=1$.

Case 5: $R(z, w(z))$ is a polynomial in $w(z)$. Then (3) can be written as

$$
w(q z)-w(z / q)+a(z) \frac{w^{\prime}(z)}{w(z)}=P(z, w(z)),
$$


where $\operatorname{deg}_{w}(P) \leqslant 4$. If $\operatorname{deg}_{w}(P)=1$, then (4) holds, and if $\operatorname{deg}_{w}(P)=0$, it follows that $R(z, w(z))$ in (3) is a polynomial of degree 0 as asserted. Now, we assume that $\operatorname{deg}_{w}(P) \geqslant 2$ and suppose that $w(z)$ has either infinitely many zeros or poles(or both). Suppose that there is a pole or a zero of $w(z)$ at $z=\hat{z}$. Note that the coefficients of (45) are rational, we can always choose a zero or pole of $w(z)$ in such way that there is no cancellation with the coefficients. Without loss of generality, suppose that there is a pole of $w(z)$ at $z=q \hat{z}$. By iterating (45), it follows that $w(z)$ has a pole of order $\operatorname{deg}_{w}(P)$, at least, at $z=q^{2} \hat{z}$, and a pole of order $\left(\operatorname{deg}_{w}(P)\right)^{2}$ at $z=q^{3} \hat{z}$, and so on. The string of poles with exponential growth in the multiplicity can not terminate. And note that the coefficients are rational and thus have finitely many zeros, and $w(z)$ has infinitely many zeros or poles, we can choose the starting point $\hat{z}$ of the iteration from outside a sufficiently large disc in such a way that no cancellation occurs. Thus

$$
n\left(K^{s}|\hat{z}|, w\right) \geqslant\left(\operatorname{deg}_{w}(P)\right)^{s-1},
$$

for all $s \in \mathbb{N}$, with $K=\max \{|q|, 1 /|q|\}$, and so

$$
\begin{aligned}
\lambda\left(\frac{1}{w}\right) & =\limsup _{r \rightarrow \infty} \frac{\log n(r, w)}{\log r} \\
& \geqslant \limsup _{s \rightarrow \infty} \frac{\log n\left(K^{s}|\hat{z}|, w\right)}{\log \left(K^{s}|\hat{z}|\right)} \\
& \geqslant \limsup _{s \rightarrow \infty} \frac{\log \left(\operatorname{deg}_{w}(P)\right)^{s-1}}{\log \left(K^{s}|\hat{z}|\right)} \\
& =\frac{\log \left(\operatorname{deg}_{w}(P)\right)}{\log K}>0 .
\end{aligned}
$$

Hence, $\rho(w) \geqslant \lambda\left(\frac{1}{w}\right)>0$, a contradiction.

Suppose that $w(z)$ has finitely many poles and zeros, then $w(z)$ is a rational function, which contradicts with our assumption.

Acknowledgements: This research was supported by the National Natural Science Foundation of China (Nos: 12001117, 12001503) and by the Basic and Applied Basic Research of Guangzhou Basic Research Program (No. 202102020438).

\section{REFERENCES}

1. Quispel GRW, Capel HW, Sahadevan R (1992) Continuous symmetries of differential-difference equations: the Kac-van Moerbeke equation and Painlevé reduction. Phys Lett A 170, 379-383.

2. Halburd R, Korhonen R (2006) Difference analogue of the lemma on the logarithmic derivative with applications to difference equations. $J$ Math Anal Appl 314, 477-487.

3. Chiang YM, Feng SJ (2008) On the Nevanlinna characteristic of $f(z+\eta)$ and difference equations in the complex plane. Ramanujan $J$ 16, 105-129.
4. Chiang YM, Feng SJ (2018) Nevanlinna theory of the Askey-Wilson divided difference operator. Adv Math 329, 217-272.

5. Chen ZX (2011) Growth and zeros of meromorphic solutions of linear difference equations. J Math Anal Appl 373, 235-241.

6. Halburd RG (2017) Elementary exact calculations of degree growth and entropy for discrete equations. Proc $R$ Soc A 473, ID 20160831.

7. Ishizaki K, Korhonen R (2018) Meromorphic solutions of algebraic difference equations. Constr Approx 48, 371-384.

8. Li S, Gao ZS (2011) Finite order meromorphic solutions of linear difference equations. Proc Japan Acad Ser A Math Sci 87, 73-76.

9. Wang Q, Han Q, Hu PC (2019) Quantitative properties of meromorphic solutions to some differentialdifference equations. Bull Aust Math Soc 99, 250-261.

10. Barnett DC, Halburd RG, Morgan W, Korhonen RJ (2007) Nevanlinna theory for the $q$-difference operator and meromorphic solutions of $q$-difference equations. Proc Roy Soc Edinburgh Sect A 137, 457-474.

11. Huang ZB, Lan ST, Zhang RR (2021) Meromorphic solutions of certain types of complex functional equations. ScienceAsia 47, 520-529.

12. Korhonen R, Wen ZT (2016) Existence of zero-order meromorphic solutions in detecting-difference Painlevé equations. Trans Amer Math Soc 368, 4993-5008.

13. Laine I, Yang CC (2007) Clunie theorems for difference and q-difference polynomials. $J$ Lond Math Soc 76, $556-566$.

14. Zhang JL, Korhonen R (2010) On the Nevanlinna characteristic of $f(q z)$ and its applications. J Math Anal Appl $369,537-544$.

15. Halburd R, Korhonen R (2017) Growth of meromorphic solutions of delay differential equations. Proc Amer Math Soc 145, 2513-2526.

16. Hayman WK (1964) Meromorphic Function, Clarendon Press, Oxford.

17. Laine I (1993) Nevanlinna Theory and Complex Differential Equations, W. de Gruyter, Berlin.

18. Hayman WK (1965) On the characteristic of functions meromorphic in the plane and of their integrals. Proc London Math Soc 14A, 93-128.

19. Mohon'ko AA, Mohon'ko VD (1974) Estimates of the Nevanlinna characteristics of certain classes of meromorphic functions, and their applications to differential equations. Siberian Math J 15, 921-934.

20. Bergweiler W, Ishizaki K, Yanagihara N (1998) Meromorphic solutions of some functional equations. Methods Appl Anal 5, 248-258.

21. Yang CC, Yi HX (1995) Uniqueness Theory of Meromorphic Functions, Science Press, Beijing.

22. Valiron G (1931) Sur la dérivée des fonctions algébroïdes. Bull de la Soc Math de France 59, 17-39.

23. Mohon'ko AZ (1971) The Nevanlinna characteristics of certain meromorphic functions. Teor Funkciŭ Funkcional Anal i Priložen 14, 83-87. [in Russian] 